\title{
An Improved Autoregressive Method with Kalman Filtering Theory for Vessel Motion Predication
}

\author{
Zhuang Lin, Qiang Yang*, Zhiqun Guo, Jun Li \\ College of Shipbuilding Engineering, Harbin Engineering University, Harbin 150001, China \\ *Corresponding author's Email: yangqiang_1986@foxmail.com
}

\begin{abstract}
It is significant and valuable to improve the time and the accuracy of ships' motion predication. Autoregressive time series analysis method $(A R)$ with Kalman filtering theory is the mainstream currently and the effectiveness for the prediction of ships' motion attitudes have been fully validated. However, the algorithm fixes the order, i.e. the length of the state vector once only, but forecasts the future data for multi-step, resulting in degradation of the step length and the accuracy, especially when the ship sails in bad sea condition. In order to solve this issue, this paper proposed a new autoregressive-multiple $(A R m)$ method, which can determine the orders and the parameters of model in real-time. The method was applied to forecast a ship's motion attitudes in eight different situations. The simulative results of autoregressive-multiple method show the validity and veracity compared with real value.
\end{abstract}

Keywords: Autoregressive-multiple; Real-time; Prediction for ship motion; Kalman fliter

\section{Introduction}

Ships will suffer the effect of the waves and swing inevitably, resulting in great security risk, particularly in the poor situation. Providing that the ship motion attitudes are able to be forecasted in several seconds, the utilization of stabilizer equipment enable ship to reduce the effect. Thereby the researches in the area of the prediction of ships' movement attitudes are pretty essential.

There are a host of studies which were carried out by domestic and foreign researchers. J .T. Fleck [1] and M. R. Bates [2] applied the stationary time series predictive method to forecast ships' motion attitudes sailing in the relative low frequency waves, and this method is able to predict 5 to 6 seconds on ships' pitching. M. S. Triantafyllou [3-6] of MIT expanded the reach in the area of the assessment of ships' motion attitudes and short-term prediction with the method of Kalman filter. In this way, the noise can be handled effectively, hence in the situation with noise; this method can predict ship rolling in 6 to 8 seconds, as well as 2 to 3 for ship pitching. Nevertheless, because of the requirement of ship state equation which is difficult to determine, when the hydrodynamic parameters and environment varies, this method is too complex to apply in engineering practice. Hongan Qiu [7] and Shuwen Zhang [8] forecasted ships' motion attitudes with period gram method and the prediction time could arrive at 5 to 7 seconds, but the consequence of prediction always has a difference of a phase angle with the truth. The maximal benefit of the time series analysis method adopted by Xiren Zhao and Xiuyan Peng $[9,10]$ is not necessary to know the prior information and voyage status. This method needs only the historical data to carry out the forecasting in 8 to 10 seconds. However, the predictive accuracy and stability still cannot meet the demand. One of the most important reasons is that the movement of ships affected by many external factors which are changing all the time. While the same order and parameters are utilized to forecast the prediction with 
a long time-step, there must be a number of mistakes, especially in the poor condition [11].

Moment estimation, which is one of the most thriving and useful preliminary methods in the time series analysis, estimates the model's parameters through the sample moments derived from the time series. The Lenvinson Recursive Algorithm [12] is effective in this area, but the sample's correlation function must be calculated accompanied by matrix inversion. Hence, Widrow's least mean square (LMS) algorithm [13] has solved this problem, which is characterized by operation simply. However, this method which is convergence slowly and grateful with the eigenvalue distribution of the data's correlation matrix is not suitable to use on the prediction online. Recursive least squares (RLS) algorithm [14] could achieve fast convergence. The algorithm, whose purpose is that the quadratic sum between expected output signal and the model output is the smallest, has been successfully applied to autoregressive time series analysis method's parameter estimation and achieved good consequences on the ship attitudes predication. However, the data adaptive filter is in terms of input data for each group, could not reflect the integrity on information. Kalman filtering algorithm is similar to the characteristics of RLS algorithm, but Kalman filtering algorithm is more optimal than RLS algorithm with the fast convergence speed.

To reduce effect of external factors on prediction of ship motion, this essay focuses on the characteristics of real-time ship motion and applies Kalman filtering algorithm and real-time model which fixes orders and parameters each step to forecast ships' motion attitude. Consequently, effects impacting on the mathematical model are eliminated. In section II, the autoregressive-multiple (ARm) method and Kalman filtering algorithm are described in detail. Then in section III, the model is utilized to forecast some future data of eight different situations.

\section{Method and Theory}

\subsection{The theory of ARm}

$A R$ model must learn the sample data $x(i), i=1,2, \ldots$ ,$n$ to determine the model's coefficients and order. Then the ends of $\widehat{p}$ sample data are used to predict future data. At the beginning, the ship heave motion sample data $x(i), i=1,2, \ldots, n$ is established. $A R$ method requires the sample data's mean must be zero mean. However, the actual ship heave data cannot meet the demand, so it is necessary to process the sample data. Calculate the mean $\bar{x}$, and then each data in the sample subtracts $\bar{x}$ and establishes the new sample. After the prediction, the results adding $\bar{x}$ are the future predictive data.

\subsubsection{Use $A R(p)$ to forecast once}

Generally, the $A R(p)$ model is:

$$
x(k)=\beta_{1} x(k-1)+\beta_{2} x(k-2)+\ldots \beta_{p} x(k-p)+e(k)
$$

In which, the time series $\{x(k), k=1,2, \ldots n\}$ is the sample data, which is considered as white noise sequence. $\{e(k), k=1,2, \ldots n\}$ is the measurement error sequence and $\left\{\beta_{i}, i=1,2, \ldots, p\right\}$ is coefficients sequence. If $k=p+1, p+2, \ldots, n$, then:

$$
\begin{gathered}
x(p+1)=\beta_{1} x(p)+\beta_{2} x(p-1)+\ldots \beta_{p} x(1)+e(p+1) \\
x(p+2)=\beta_{1} x(p+1)+\beta_{2} x(p)+\ldots \beta_{p} x(2)+e(p+2) \\
\vdots \\
x(n)=\beta_{1} x(n-1)+\beta_{2} x(n-2)+\ldots \beta_{p} x(n-p)+e(n)
\end{gathered}
$$

And, if

$$
\begin{aligned}
X & =[x(p+1), x(p+2), \ldots, x(n)]^{T} \\
\Phi & =\left[\begin{array}{cccc}
x(p) & x(p-1) & \ldots & x(1) \\
x(p-1) & x(p) & \ldots & x(2) \\
\vdots & \vdots & \vdots & \vdots \\
x(n-1) & x(n-2) & \ldots & x(n-p)
\end{array}\right] \\
e & =[e(p+1), e(p+2), \ldots, e(n)]^{T} \\
B & =\left[\beta_{1}, \beta_{2}, \ldots, \beta_{p}\right]^{T}
\end{aligned}
$$

The equation (1) can be written as vector equations in the form:

$$
X=\Phi B+e_{0}
$$

Using recursive least squares method, we can estimate $\beta_{i}, i=1,2, \ldots, p$, and at the same time, get the optimal order $\widehat{p}$ using $A I C$ criterion. Then establish the optimal $A R(p)$ model (8) and calculate a forecasting with $x(i), i=1,2, \ldots, n$

$$
\widehat{x}(n+1)=\sum_{j=1}^{p} \widehat{\beta}_{j} x(n-j+1)
$$

\subsubsection{Vary the Sample Data and Predict $l$ Step}

On the fundament of $A R(p)$ method, we build a circle (Figure 1). Put the forecasting data $\widehat{x}(n+l)(l<L$, $L$ is the max predictive step) into the sample data, and establish a new sample data $x(i), i=1,2, \ldots, n+l . \widehat{p}_{l}$ is the optimal order for the $l$ step.

Calculate the function $\operatorname{AIC}(1), \operatorname{AIC}(2), \ldots, A I C(M)$ ( $M$ is the maximum order), meanwhile account the square sum of residual errors $\left(S_{p}(n+l)\right)$. Where

$$
S_{p}(n+l)=\left(X-\Phi_{N+l} \widehat{B}_{n+l}\right)^{T}\left(X-\Phi_{N+l} \widehat{B}_{n+l}\right)
$$




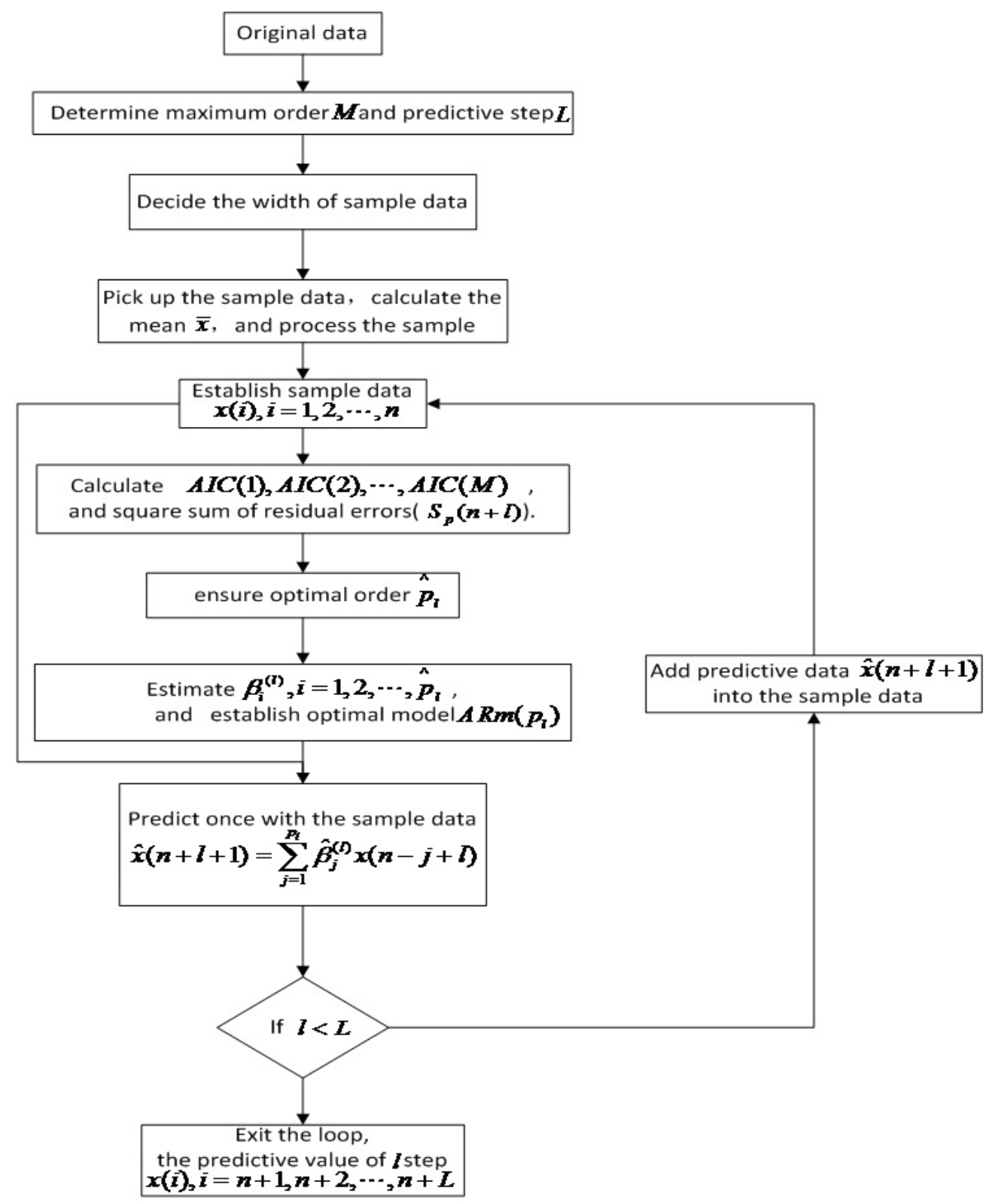

Figure 1 Circle of autoregressive-multiple 


$$
\begin{array}{r}
A I C\left(p_{l}\right)=\ln \left(S_{p}(n+l) /(n+l)\right)+2 p_{l} /(n+l) \\
\left(p_{l}=1,2, \ldots, M\right)
\end{array}
$$

After that, compare the size of $\operatorname{AIC}\left(p_{l}\right)$, and when $p_{l}=\widehat{p}_{l}$, there is

$$
\operatorname{AIC}\left(\widehat{p}_{l}\right)=\min \left\{\operatorname{AIC}\left(P_{l}\right)\right\}
$$

Then utilize recursive least squares method equation (12) to estimate.

$$
\begin{aligned}
\widehat{B}_{n+l+1}= & \widehat{B}_{n+l}+k(n+l+1)\left[x^{\prime}(n+l+1)\right. \\
& \left.-\Phi_{n+l+1}^{T} \widehat{B}_{n+l}\right]
\end{aligned}
$$

Where, $k(n+l+1)$ is time-varying gain matrix, and

$$
\begin{aligned}
k(n+l+1)= & \frac{P_{n+1} \Phi_{n+l+1}}{1+\Phi_{n+l+1}^{T} P_{n+l} \Phi_{n+l+1}} \\
& \left(P_{n+l}=\left[\Phi_{n+l}^{T} \Phi_{n+l}\right]^{-1}\right)
\end{aligned}
$$

At the last, $A R m\left(P_{l}\right)$ model equation (14) is determined, and we can utilize $A R m\left(P_{l}\right)$ model to carry out the $l+1$ step prediction with $x(i), i=1,2, \ldots, n+l$.

$$
\widehat{x}(n+l+1)=\sum_{j=1}^{p_{l}} \widehat{\beta}_{j}^{(l)} x(n-j+l)
$$

Put the prediction data into the sample data, while $l+1=L$, the program exit the loop and $x(i), i=n+$ $1, n+2, \ldots, n+L$ are the predictive value.

\subsection{The principle of Kalman filter and state esti- mation}

$\beta(t)$ is the discrete linear system state vector which is m-dimensional at time $t$, and $x(t)$ is the system observed data vector in the same time which is n-dimensional. So the state-space model of system can be expressed as following

Equation of state:

$$
\beta(t+1)=\Phi(t+1, t) \beta(t)+v_{1}(t)
$$

Equation of observed:

$$
x(t)=c(t) \beta(t)+v_{2}(t)
$$

In which, $\Phi(t+1, t)(m \times m)$ is the State transition matrix; $v_{1}(t)(m \times 1)$ is the process noise vector which is zero-mean white noise process, whose correlation matrix is $Q_{1}(t) ; c(t)(n \times m)$ is known as the observation matrix; $v_{2}(t)(n \times 1)$ is observe noise vector, which is zero-mean white noise too, and the correlation matrix of $v_{2}(t)$ is $Q_{2}(t)$. As well, $v_{1}(t)$ and $v_{2}(t)$

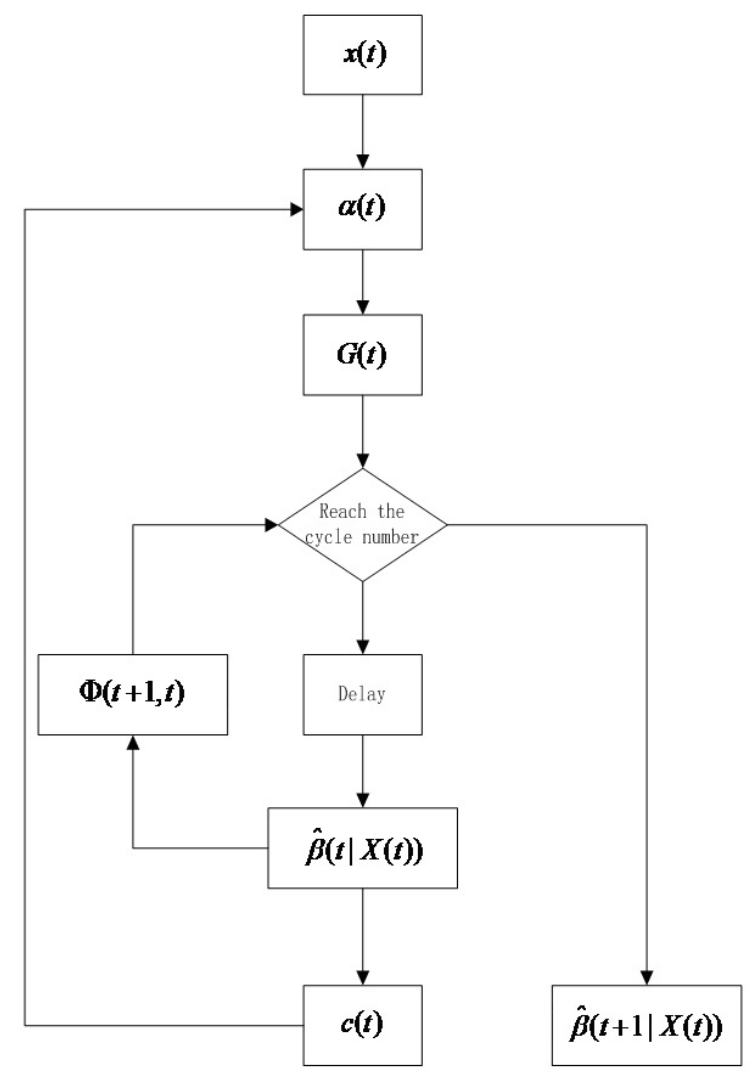

Figure 2 Kalman filter flow chart

are counted respectively, we can get the $E\left[v_{1}(t) v_{2}^{T}(k)\right]=$ 0 for all of $t$ and $k$ by another way.

If $\widehat{\beta}(t+1 \mid X(t))$ and $\widehat{\beta}(t \mid X(t))$ are the minimum variance estimation step prediction and filter estimates of state vector at time $t$ respectively which are obtained from the observe data vector $x(1), x(2), \ldots, x(t)$, in which $X(t)$ is the vector composed by Vector of past observations data $x(1), x(2), \ldots, x(t) . \widehat{x}(t \mid X(t-1))$ is the minimum variance forecast came from the reaction of all the previous measurement data vector from 1 to $t-1$. So there are the expression as following based on the first prediction algorithm.

New interest rate process:

$$
\alpha(t)=x(t)-\widehat{x}(t \mid X(t-1))
$$

Kalman gain

$$
\begin{array}{r}
G(t)=\Phi(t+1, t) K(t, t+1) \cdot c^{T}(t) . \\
{\left[c(t) K(t, t+1) c^{T}(t)+Q_{2}(t)\right]^{-1}}
\end{array}
$$

First predication

$$
\begin{aligned}
\widehat{\beta}(t+1 \mid X(t))= & \Phi(t+1, t) \widehat{x}(t \mid X(t-1)) \\
& +G(t) \alpha(t)
\end{aligned}
$$

State estimation

$$
\widehat{\beta}(t \mid X(t))=\Phi(t+1, t) \widehat{x}(t \mid X(t-1))
$$


State filtering error correlation matrix

$$
K(t)=K(t, t+1)-\Phi(t+1, t) G(t) K(t, t-1)
$$

State prediction error correlation matrix

$$
\begin{array}{r}
K(t+1, t)=\Phi(t+1, t) K(t, t+1) \Phi^{T}(t+1, t) \\
+Q_{1}(t)
\end{array}
$$

\subsection{Kalman filter algorithm used in adaptive $A R m(p)$ Model parameter estimation}

\subsubsection{State space model}

The Arm model can be thought of a once linear minimum variance prediction. The input vector is set up by $x(t-1), \ldots, x(t-p)$ which has been known. Based the weight between input vector and parameter $\beta(t)$, $x(t)$ which is expected can be estimation. Based the basic principle of wiener filter, the optimal parameter estimation of Arm model is equivalent to the optimal solution $\widehat{\beta}_{0}(t)$ of wiener filter at time $t$.

Considering that the optimal parameters change over time, so parameter estimation process is seen as nonstable process and introduces the process noise $v(t)$ :

$$
\widehat{\beta}_{0}(t+1)=\widehat{\beta}_{0}(t)+v(t)
$$

The use of adaptive Kalman filtering algorithm Arm online estimate model parameters, the model assumes that the state of Arm is a random walk model.

When the adaptive Kalman filter algorithm estimates the parameters of ARm model, the state of Arm is a random walk model (Figure 2):

$$
\beta(t+1)=\beta(t)+v(t)
$$

$v(t)$ always is zero-mean stationary random process and the correlation matrix is $Q(t)=E\left(v(t) v^{T}(t)\right]=q I$ where $q$ is scalar and the variance of $v(t)$. It is obvious that the transition matrix is the unit matrix, and the observation equation is:

$$
x(t)=\beta(t) X^{T}(t)+\varepsilon(t)
$$

In which: $X^{T}(t)=|x(t-1), x(t-2), \ldots, x(t-p)|$ is measurement matrix, the mean of $\varepsilon(t)$ is zero and the variance is $\delta_{\varepsilon}^{2} .|v(t)|$ and $\varepsilon(t)$ are independence to each other.

When the time of iteration tends to the infinity, the parameter of Kalman filter algorithm is equivalent to the optimal solution of Wiener.

\subsubsection{Derivation of adaptive Kalman filtering al- gorithm in the non-stationary situation}

$\widehat{\beta}(t)=\widehat{\beta}\left(t \mid x_{t}\right)$ is the parameter vector estimation composed by the observation data $x(1), x(2), \ldots, x(t)$, according to the recurrence formula of Kaman filter algorithm(17)(18), we can get

$$
\begin{gathered}
\beta(t)=\beta(t-1)+g(t) \alpha(t) \\
g(t)=K(t-1) X(t)\left[X^{T}(t) K(t-1) X(t)-\sigma_{\varepsilon}^{2}\right]^{-1}
\end{gathered}
$$

In which New interest rate process

$$
\alpha(t)=x(t)-X^{T}(t) \widehat{\beta}(t-1)
$$

is scalar, Kalman gain $g(t)(p \times 1)$ is vector.

State filtering error in the state space model of (23)(24) is definite $e(t)$, the state prediction error is $K(t+1, t)$, so from $(16)(20)(21)$,we can see:

$$
\begin{aligned}
& e(t)=\beta(t)-\widehat{\beta}\left(t \mid x_{t}\right) \\
& K(t+1, t)=K(t)+Q(t)=K(t)+q I
\end{aligned}
$$

In which:

$$
K(t)=K(t, t-1)-g(t) X^{T}(t) K(t, t-1)
$$

Equations (9) (10) compose the state estimation process of adaptive Kalman filter algorithm. Using the above formula the adaptive ARm model can be established for the non-stationary processes, its parameter fluctuate around the mean. According to the processes we must assume:

1 , the variance of $\varepsilon(t)$ is $\sigma_{\varepsilon}^{2}$

2 , the variance of $v(t)$ is $q$ (in this paper $q=10^{-4}$ )

Obviously $\sigma_{\varepsilon}^{2}$ cannot be obtained with a precise mathematical model, However, in most cases output Signal to Noise Ratio from the adaptive filter is usually in very high. So when we calculate the $g(t)$, we can see 0.001 or 0.01 multiplies $|x(t), t=p+1, p+2, \ldots, n|$ as the variance $\sigma_{\varepsilon}^{2}$.

If the state transition matrix of the state equation is known, then the state space method could have a good description of non-stationary, but it cannot predict the actual transition matrix, so the use of random walk in the above non-steady state model gives a simple case functional description (especially slow time-varying case).

The impact of Non-stationary conditions for the algorithm is basically reflected in the correlation matrix $Q(t)$, while he decided the recursive correction. In addition to the above form $Q(t)=E\left(v(T) v^{T}(t)\right]=q I$, you can also take $Q(t)=q K(t)$. 
In this situation the recursive formula of Kalman filter algorithm can be expressed as following:

$$
\begin{aligned}
g(t) & =K(t-1) X(T)\left[X^{T}(t) K(t-1) X(T)\right. \\
\left.-\xi_{\min }\right]^{-1} & \\
\alpha(t) & =x(t)-X^{T}(t) \widehat{\beta}(t-1) \\
\beta(t) & =\beta(t-1)+g(t) \alpha(t) \\
K(t)=(1+q)\left[K(t, t-1)-g(t) X^{T}(t) K(t, t-1)\right] & \\
\xi_{\min } & =\sigma_{\varepsilon}^{2} /(1+q)
\end{aligned}
$$

\section{Instances of the ship heave motion predic- tion}

In this paper, we adopted the actual measured data when a ship sailed in eight different situations ( 0 -Heave2,0-Heave-4,0-Pitch-2,0-Pitch-4,90-Heave-2,90-Heave4,90-Roll-2 and 90-Roll-4 where the first number is angle of the wave and the last number is height of the wave ). There are 220 data in each sample, and of which the first 200 data are used for model establishment as well as the other 20 data for forecast verification. MATLAB programming language was used to predict the ship's motion attitudes in the future $10 \mathrm{~s}$ $(L=20)$. Forecasting performance relative square error is

$$
J F=\frac{\sqrt{\frac{1}{2} \sum_{l=1}^{L}[\widehat{x}(n+l)-x(n+l)]^{2}}}{|x|_{\max }} \times 100 \%
$$

Where, $l$ is the predictive step.

Table 1 The orders of $A R(p)$ and $A R m(p)$

\begin{tabular}{|c|c|c|c|c|c|c|c|c|c|c|}
\hline Step & $\mathbf{1}$ & $\mathbf{2}$ & $\mathbf{3}$ & $\mathbf{4}$ & $\mathbf{5}$ & $\mathbf{6}$ & $\mathbf{7}$ & $\mathbf{8}$ & $\mathbf{9}$ & $\mathbf{1 0}$ \\
\hline$A R(P)$ & 7 & 7 & 7 & 7 & 7 & 7 & 7 & 7 & 7 & 7 \\
\hline$A R m\left(P_{l}\right)$ & 3 & 7 & 3 & 26 & 7 & 7 & 7 & 7 & 9 & 7 \\
\hline Step & $\mathbf{1 1}$ & $\mathbf{1 2}$ & $\mathbf{1 3}$ & $\mathbf{1 4}$ & $\mathbf{1 5}$ & $\mathbf{1 6}$ & $\mathbf{1 7}$ & $\mathbf{1 8}$ & $\mathbf{1 9}$ & $\mathbf{2 0}$ \\
\hline$A R(P)$ & 7 & 7 & 7 & 7 & 7 & 7 & 7 & 7 & 7 & 7 \\
\hline$A R m\left(P_{l}\right)$ & 11 & 8 & 4 & 7 & 7 & 7 & 7 & 7 & 4 & 4 \\
\hline
\end{tabular}

Table 1 shows the orders of both $A R$ and $A R m$ to forecast "0-Heave-2" (the angle between the wave and the vessel is $0^{\circ}$ and the height of the wave is $2 \mathrm{~m}$ ) for 20 steps (the maximum order is $M=30$ ). We can see clearly $A R(P)$ use the same order when $A R m\left(P_{l}\right)$ use the real-time orders. According to the formulas (8) and (14), we can know the model of $A R(P)$ is

$$
x(n+l)=\sum_{i=1}^{7} \beta_{i} x(n+l-i)+e(n) \quad l \leq L
$$

And $A R m\left(P_{l}\right)$ model is

$$
x(n+l)=\sum_{i=1}^{p_{l}} \beta_{i}^{(l)} x(n+l-i)+e(n) \quad l \leq L
$$

Figure 3-10 (num1-motion-num2, num1 is the angle between the wave and the vessel, num2 is the height of the wave) are the comparison of forecast results of $\mathrm{AR}$, and the forecast results of $A R m$ and the real value in each situation. From these figures, it can be seen that the predictive consequences of $A R m$ are better than predictive consequences of AR, especially in the peak of waves. Table 2 is the predictive performance of both AR method and Arm method, when the forecasting time-step is $5 \mathrm{~s}, 7 \mathrm{~s}$ and $10 \mathrm{~s}$. It can be seen that the error of AR is increasing as the increasing of timestep, especially exceeded 14 steps (over 7s). But the error from $A R m$ is relatively stable. In the nutshell, the validity of $A R m$ is much better than AR. Comparing the results in 2 meter waves and 4 meter waves, the precision of AR is declined clearly, however, the precision of $A R m$ is still stable.

Table 2 The prediction in 5s, 7s and $10 \mathrm{~s}$

\begin{tabular}{|c|c|c|c|c|}
\hline situation & method & $\mathbf{5 s}$ & $\mathbf{7 s}$ & $\mathbf{1 0 s}$ \\
\hline \multirow{2}{*}{ 0-Heave-2 } & $A R(p)$ & $7.47 \%$ & $14.59 \%$ & $22.52 \%$ \\
\cline { 2 - 5 } & $A R m\left(p_{l}\right)$ & $5.58 \%$ & $5.01 \%$ & $4.46 \%$ \\
\hline \multirow{2}{*}{ 0-Heave-4 } & $A R(p)$ & $12.06 \%$ & $15.91 \%$ & $28.67 \%$ \\
\cline { 2 - 5 } & $A R m\left(p_{l}\right)$ & $1.40 \%$ & $3.62 \%$ & $6.79 \%$ \\
\hline \multirow{2}{*}{ 0-Pitch-2 } & $A R(p)$ & $13.93 \%$ & $23.47 \%$ & $37.28 \%$ \\
\cline { 2 - 5 } & $A R m\left(p_{l}\right)$ & $4.79 \%$ & $11.87 \%$ & $11.06 \%$ \\
\hline \multirow{2}{*}{ 0-Pitch-4 } & $A R(p)$ & $12.41 \%$ & $14.58 \%$ & $29.99 \%$ \\
\cline { 2 - 5 } & $A R m\left(p_{l}\right)$ & $12.06 \%$ & $11.93 \%$ & $15.13 \%$ \\
\hline \multirow{2}{*}{ 90-Heave-2 } & $A R(p)$ & $21.67 \%$ & $25.51 \%$ & $32.83 \%$ \\
\cline { 2 - 5 } & $A R m\left(p_{l}\right)$ & $7.86 \%$ & $14.20 \%$ & $19.58 \%$ \\
\hline \multirow{2}{*}{ 90-Heave-4 } & $A R(p)$ & $12.37 \%$ & $14.27 \%$ & $20.09 \%$ \\
\cline { 2 - 5 } & $A R m\left(p_{l}\right)$ & $6.15 \%$ & $5.52 \%$ & $6.84 \%$ \\
\hline \multirow{2}{*}{ 90-Roll-2.0 } & $A R(p)$ & $8.54 \%$ & $11.85 \%$ & $11.70 \%$ \\
\cline { 2 - 5 } & $A R m\left(p_{l}\right)$ & $4.69 \%$ & $5.14 \%$ & $5.91 \%$ \\
\hline \multirow{2}{*}{ 90-Roll-2.0 } & $A R(p)$ & $24.53 \%$ & $29.18 \%$ & $33.19 \%$ \\
\cline { 2 - 5 } & $A R m\left(p_{l}\right)$ & $4.24 \%$ & $4.37 \%$ & $12.01 \%$ \\
\hline
\end{tabular}

\section{Conclusion}

This paper improved $A R$ method and proposes autoregressive-multiple predictive method $A R m$. The improved method can fix the orders and parameters in a real time and reduce the effect from the external factors impacting on the ship motion. Through simulative results in eight different situations, we can see this method can effectively predict all ship motions in 8 to 10 s. Comparing with $A R$ method the precision of 


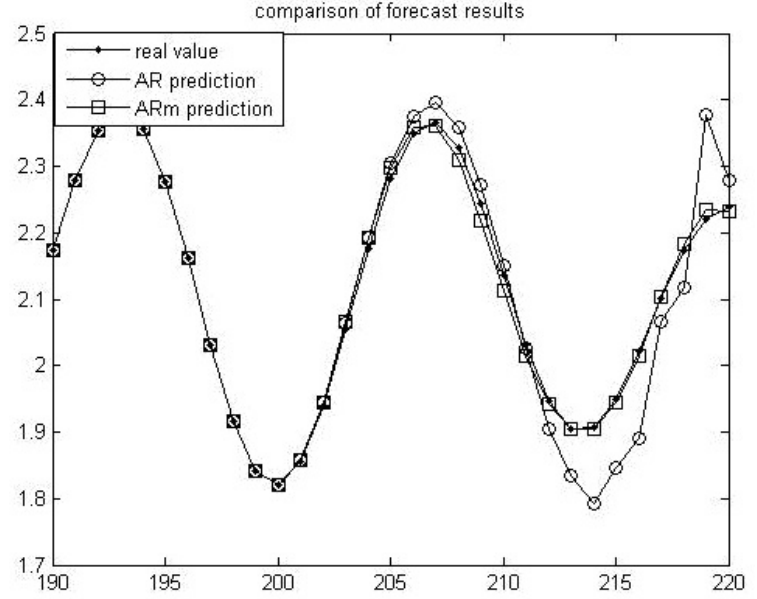

Figure 3 0-Heave-2

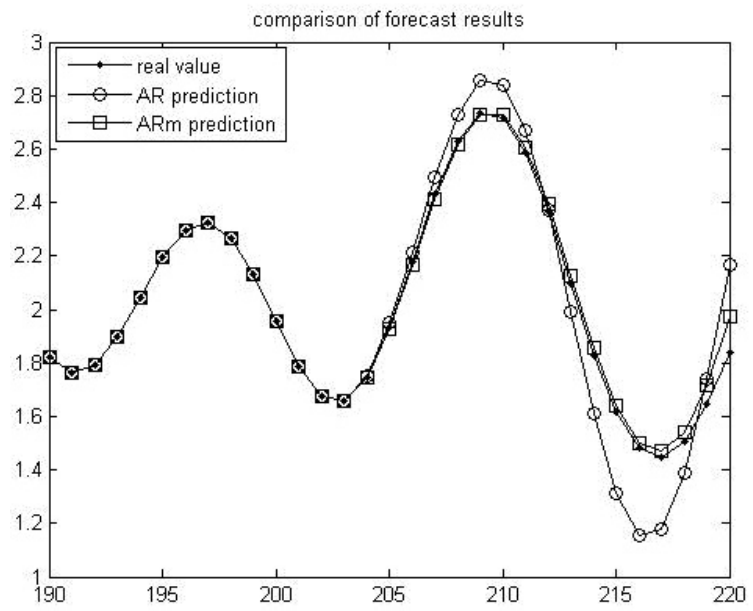

Figure 4 0-Heave-4

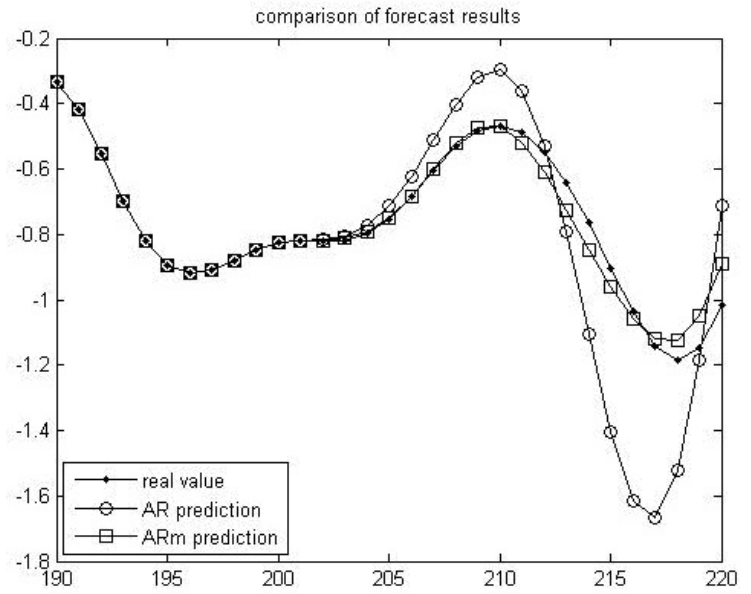

Figure 5 0-Pitch-2

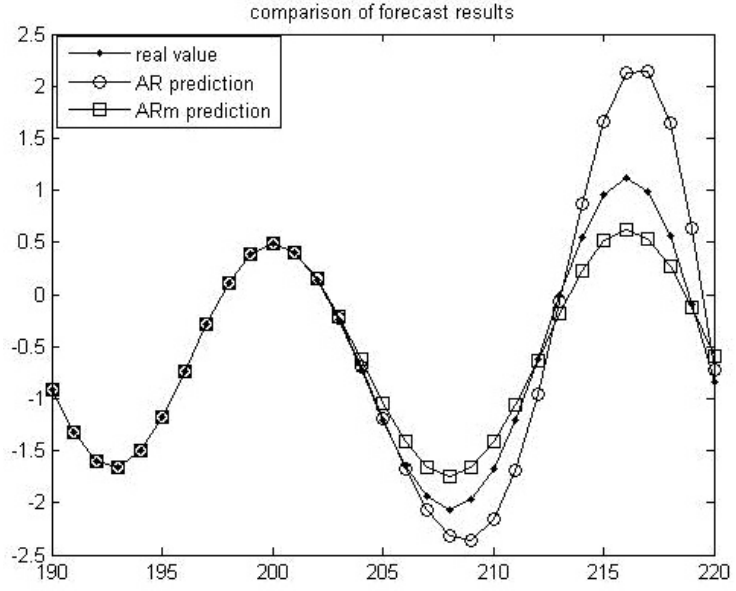

Figure 6 0-Pitch-4

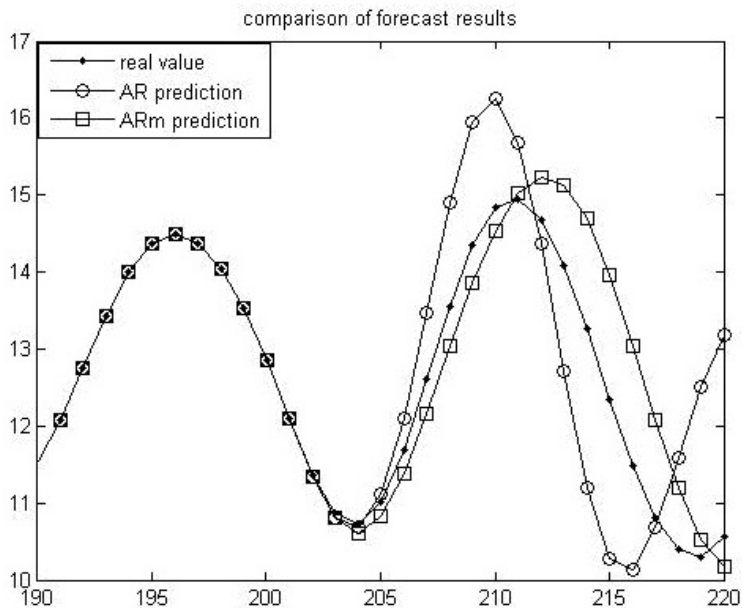

Figure 7 90-Heave-2

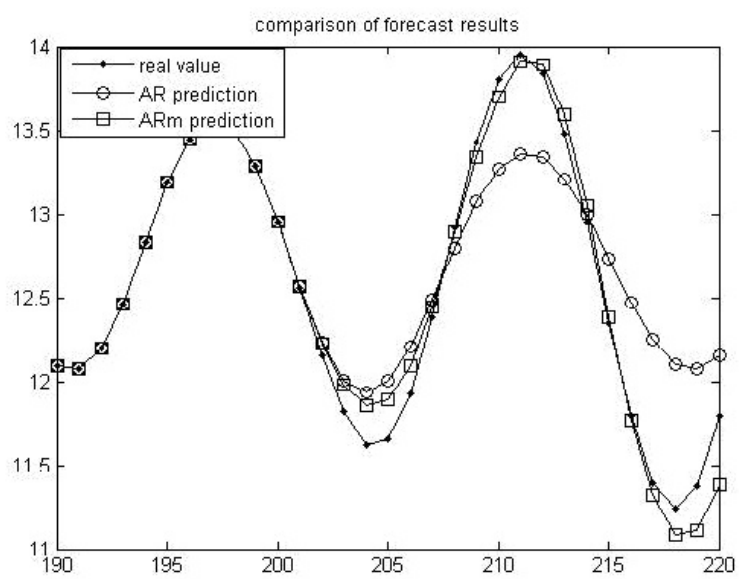

Figure 8 90-Heave-4 


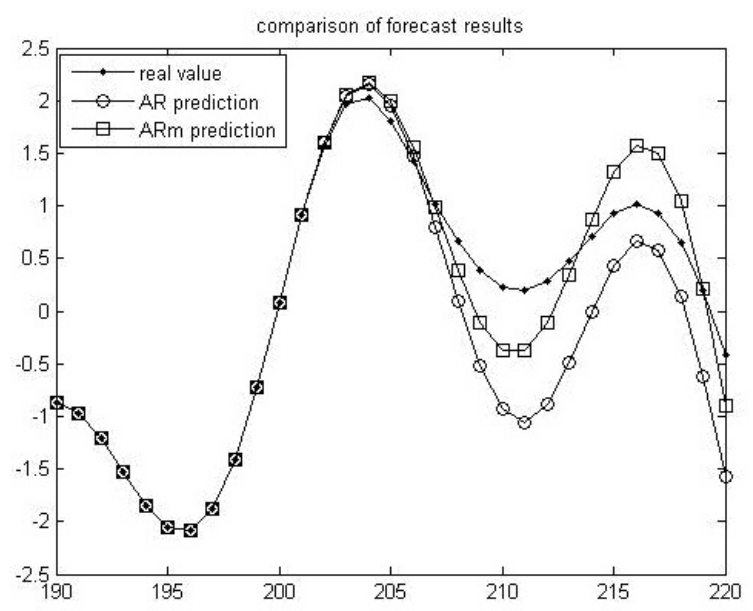

Figure 9 90-Roll-2

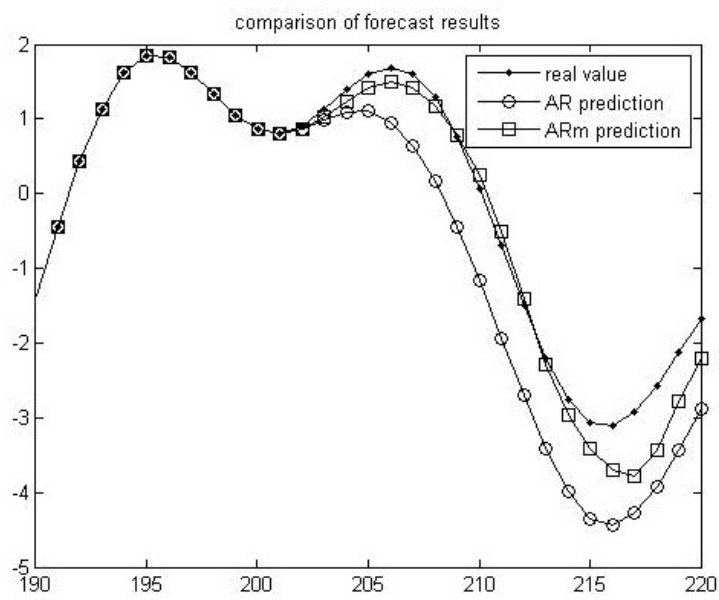

Figure 10 90-Roll-4

$A R m$ method is more veracious and stable, especially in the 4 meter waves. The method is universal and can be applied to all degrees of freedom ship motion predictions, such as the ship rolling, pitching and so on, so there is essential signification in ship engineering.

\section{Acknowledgment}

This work is supported by the Fundamental Research Funds for the Central Universities under Grant HEUCFR1001 and the Special Funds of the National Natural Science Foundation of China. Grant No. 60927008

\section{References}

[1] Fleck, J.T., "Short Time Predction of the Motion of a Ship in Waves", Proc.Ist Conf. On Ships and Waves, October 1954, Published by Council on Wave Research and SNAME.

[2] Bates, M.R., Book, D.H., and Poweel, F.D., "Ana$\log$ Computer Application in Predictor Design", IRE
Trans. On Elec. Com., Sep., Vol.EC-6, No.65-22, 1965.

[3] Triantafyllou, M., and Bodson, M., "Real Time Prediction of Marine Vessel Motion Using Kalman Filtering Techiques”, Proc. OTC, Houston, Texas, 1982.

[4] Triantafyllou, M., "Real Time Prediction and Estimation of Ship Motion Using Kalman Filtering Techiques", NASA-CT-169284, No.82-31637, 1982.

[5] Triantafyllou, m., Athans, M., "Real Time Estimation of Motions of a Destroyer Using Kalman Filtering Techiniques", Laboratory for Information and Decision Systems Rep., MIT Cambridge. MAY, 1983.

[6] Triantafyllou, M., Athans, M., "Real Time Estimation of The Heaving and piching Motions of a Ship Using a Kalman Filter", Proc. Oceanics 81, Boston MA, Sept. 1981.

[7] Qiu Hongan, "Establishing and Simulation for Random Ocean State Model", Journal of System Simulation Vol.12, No.3, pp.226-228, 2000.

[8] Zhang Shuwen, "A Comparative Study of Sequency Spectrum with Frequency Spectrum of Random Sea Waves I". The Sequency Spectral Method of Random Sea Waves, Transactions of Oceanology and Limnology, No.1, pp.1-6, 1999.

[9] Zhao Xiren, Peng Xiuyan, Shen Yan, "Study Status Quo of Extremely Short-Time Modeling and Predicting of Ship Motion", Ship Enineering, No.3, pp.4-8, 2002.

[10] Peng Xiuyan, "On-line Prediction and Simulation Tchnique Research on Ship Motion Attitude", Dissertation for the Degree of D.Eng, 2006.

[11] Ma Jie, Han Yuntao, Li Guobin, "Prediction of ship pitching motion based on AR method", Ship Science and Technology, Vol.28, No.3, Jun. 2006.

[12] Weiqin, Yang, "The Time Series Analysis and Dynamic Data Modeling", Beijing Institute of Theconology Press, 1988.

[13] Dini, "Adaptive Filtering Algorithm and Achieve", Electronics Industry Press, 2004.

[14] Peng Xiuyan, Zhao Xiren, "Ship Attitude Motion on Real-time Forecasting Algorithm”, Journal of System Simulation, vol.12, No.1, 2007.

[15] Peng Xiuyan, "Adaptive Estimation Method of AR Model Parameters", Jouranal of Harbin Institute of Tehnology, Vol.141, No.19, Sep. 2009. 